\title{
The High Expression of CD276/HAVCR2 and CD163 is an Adverse Immune Subtype of Glioblastoma and is Closely Related to Epithelial-Mesenchymal Transition
}

\section{Xiaohong Hou}

Affiliated Hospital of Zunyi Medical College

Guiyin Zhou

Affiliated Hospital of Zunyi Medical College

Yinchun Fan

Affiliated Hospital of Zunyi Medical College

Qiang Zhang

Affiliated Hospital of Zunyi Medical College

Chengming Xiang

Affiliated Hospital of Zunyi Medical College

Fang Cao

Affiliated Hospital of Zunyi Medical College

Shengtao Yao ( $\square$ yst@zmu.edu.cn )

Affiliated Hospital of Zunyi Medical College https://orcid.org/0000-0002-6564-2261

Primary research

Keywords: CD276, CD163, epithelial-mesenchymal transition (EMT), Glioblastoma (GBM), HAVCR2

Posted Date: May 28th, 2020

DOI: https://doi.org/10.21203/rs.3.rs-31174/v1

License: (c) (1) This work is licensed under a Creative Commons Attribution 4.0 International License.

Read Full License 


\section{Abstract}

\section{Background}

Glioblastoma (GBM) is one of the most malignant tumors that can afflict the central nervous system. Previous studies have observed that there are individual differences in the treatment response of immune checkpoint inhibitors in glioblastoma. This study's aim is to ascertain the factors that may affect the efficacy of immunosuppressant therapy.

\section{Methods}

The clinical data of this study were obtained from a public database. Then, the data was analyzed and processed by $\mathrm{R}$ software and corresponding $\mathrm{R}$ package. To verify the results of the analysis, information was gathered from $89 \mathrm{GBM}$ patients in our hospital and thereafter the corresponding paraffin sections were stained and quantitatively analyzed by immunohistochemistry.

Results

From the analysis, it was observed that both CD276 and HAVCR2 were significantly overexpressed in GBM and could be associated with patient prognosis. The analysis of single cell RNA sequencing data and GBM data analysis found an immune subtype with poor prognosis. Further analysis found that the high expression of CD276, HAVCR2 and CD163 was closely related to epithelial-mesenchymal transition (EMT) and could affect the patient prognosis of PD-L1 high expression. GSVA enrichment analysis showed that CD276, HAVCR2 and CD163 might induce EMT by JAK-STAT3 signaling pathway, and RUNX1 and IKZF1 might be transcription factors that regulate CD276/HAVCR2 high expression.

\section{Conclusions}

We found an immune subtype with poor prognosis of GBM, the high expression of CD276, HAVCR2 and CD163 with EMT are closely related and may be one of the factors affecting the efficacy of Anti-PD-L1.

\section{Background}

Glioblastoma (GBM) is a malignant tumor of the central nervous system. The main treatment methods are surgery, adjuvant radiotherapy and chemotherapy. Despite the active treatment, GBM exhibits characteristics such as short median survival, high recurrence rate, poor prognosis, etc. [1-4]. The primary method of adjuvant treatment after operation is to administer chemotherapy. However, the individual effect of chemotherapy is poor [5-7]. The low efficacy of traditional tumor treatment programs have prompted researchers to actively seek more effective and targeted treatment options. After active exploration, the researchers found a research direction that may change the current therapeutic effect: immune checkpoint inhibitors. However, the therapeutic effect is still not satisfactory to us, and it is critical to find the potential factors that affect the curative effect. 
Normally, when the body has an inflammatory response, the main function of the immune checkpoint is to reduce the molecules involved in the inflammatory response in the body and maintain immune homeostasis, thereby preventing the autoimmune response. But the tumor cells use this mechanism to achieve immune escape [8]. Therefore, the study of immune checkpoint is crucial. Immune checkpoint inhibitors primarily activate the body's immune response by inhibiting immune checkpoint gene surface ligands, thus affecting the development and progression of tumors. At present, the most studied immune checkpoint in GBM is PD-1 or PD-L1 [9-12], and the current GBM immune checkpoint inhibitors, mainly targeting PD-1 or its ligand, PD-L1[12-14], have been used in clinic to improve the prognosis of GBM patients [15]. Some studies have shown that for GBM patients, the combination of immune checkpoint inhibitors and other drugs is more effective than treatment alone [16-20]. But previous studies have also observed that there are individual differences in the treatment of immune checkpoints and thus is effective only for some patients [11], and studies of other immune checkpoints have been poorly reported. This indicates the need to dispense personalized treatment as per a patient's condition during the treatment process. Hence, it is necessary to explore the possible causes of individual differences in treatment and other effective immune checkpoint genes and subtypes with good prognosis, so that the personalized treatment of GBM is made possible.

Epithelial-mesenchymal transition (EMT) plays an important role in tumor invasion, metastasis and so on, and is closely related to its related inducers, transcription factors, signaling pathway genes and tumorassociated macrophages (TAMS), and TAMS can induce EMT [21, 22]. Most of the immune cells in brain tumors are macrophages. TAMS are considered to be supportive matrix of tumor growth and are generally considered to be associated with CD163, CD206, etc. [23, 24]. Most TAMS are considered to exhibit a M2 phenotype, whereas M2 macrophages can produce cytokines such as IL-10, IL- 6 and TGF- $\beta$, which contributes to immunosuppression and thus facilitates tumor growth and induce EMT [22, 24, 25]. CD163 is primarily expressed by monocytes/macrophages and is a marker of M2-type macrophages. In tissues, CD163 is mainly resident in tissue macrophages [26], and CD163 is considered the most specific marker of TAMS $[27,28]$. Recent studies have reported that CD163 can promote the occurrence of glioma through CK2 and may be a potential new target for glioma treatment $[29,30]$, and closely related to EMT in other cancers [31, 32]. But the association and function of CD163 with immune checkpoint genes and EMT in GBM is yet to be examined.

For the immune checkpoint genes in this study, previous researches only found CD276 to be associated with GBM prognosis $[33,34]$. HAVCR2 was also found to be associated with adaptive immune cells, which can affect inflammatory response and also affect the prognosis of GBM [17, 35, 36]. This study combined multi-database to study the relationship of them, thus ultimately identifying the high expression of CD276, HAVCR2 and CD163 may be one of the factors affecting the efficacy of Anti-PD-L1 by induce epithelial-mesenchymal transition.

\section{Materials And Methods}

\section{Data source}


In this study, the RNA expression data and clinical data of GBM patients was obtained from The Cancer Genome Atlas (TCGA) ( $n=168)$ (http://www.tcga.org/) database, Chinese Glioma Genome Atlas (CGGA) $(n=138)$ (http://www.cgga.org.cn/) database, and single cell RNA sequencing data of GBM patients from Gene Expression Omnibus (GEO) (https://www.ncbi.nlm.nih.gov/geo/) database: GSE84465(4 patient samples), GSE 103224(8 patient samples). While the immune and stromal scores were acquired from the ESTIMATE algorithm, the expression data of immune cells were obtained from TIMER database (https://cistrome.shinyapps.io/timer/). The pathway data for GSVA enrichment analysis was taken from the MsigDB database (https://www.gsea-msigdb.org/gsea/msigdb/). To verify the analysis results of this study, 89 cases of pathological results of the past 5 years were collected from the affiliated hospital of the Zunyi medical university. This consisted of high-grade glioma patients clinical information and corresponding paraffin tissue sections. All patients were diagnosed by two independent neuropathologists as per the World Health Organization classification guidelines. This study was approved by the ethics committee of the affiliated hospital of the Zunyi medical university. To find upstream transcription factors (TFs) that may regulate CD276/HAVCR2, we jointly UCSC (https://xenabrowser.net/datapages/), JASPAR (http://jaspar.genereg.net) and CISTROME (http://cistrome.org/) databases for analysis.

\section{Data Processing}

Patients whose survival data was missing were excluded from this study. This reduced the dimensionality of the data of numerical variables to two categories of data based on the best cut-off value. Initially the heatmap analysis and the survival curve of immune checkpoint genes were undertaken. The expression of CD276 and HAVCR2 in each grade of glioma was analyzed in GEPIA (http://gepia.cancer-pku.cn/) database. To further determine the expression of genes in macrophages, TSNE clustering and data processing were performed on GSE84465 and GSE103224 dataset.

Simultaneously, the survival curve and infiltration was plotted through the expression data of immune cells in the TIMER database to accurately comprehend the effect of immune cells on the prognosis of GBM patients. The correlation between CD276/HAVCR2 and CD163 was further assessed through the application of the Pearson correlation analysis. Then CD163, CD276 and HAVCR2 were regrouped as per the results through the cut-off best truncation values. To grasp the possible biological processes involved in TAMS, CD276 and HAVCR2, the GSVA enrichment analysis was performed.

\section{EMT Related Genes Analysis And Lasso Risk Model Construction}

We analyzed the relationship between I-IV group and EMT related genes by $T$ test and analysis of Variance. We further calculated the EMT risk score of each patient by using the Lasso algorithm using genes related to the prognosis of patients, and then divided the patients into high or low risk group. 


\section{Immunohistochemistry}

Immunohistochemistry was utilized to detect the protein expression levels of CD163, CD276 and HAVCR2. We collected the corresponding paraffin pathological sections of the patients. Anti-CD163, CD276 and HAVCR2 antibodies were used at a dilution of 1:200. Each stained section was reviewed and evaluated by two independent pathologists. The expression of the protein was quantified by Image-pro Plus 6.0 software. The quantitative formula was protein expression $=$ IOD/Area.

\section{Statistical analysis}

All statistical analyses were undertaken using the R software (3.6.1). Statistical methods such as Cox regression analysis, Univariate variance analysis, Pearson correlation analysis, Log-rank test, $T$ test,Analysis of Variance and Logistic regression analysis were applied. For all statistical methods, $\mathrm{P}<$ 0.05 was considered statistically significant.

\section{Results}

\section{Identification of immune checkpoint genes CD276 and HAVCR2}

To identify the immune checkpoint genes of this study's focus, it was observed that CD276, HAVCR2 and CD47 genes were significantly overexpressed in most tumors, including GBM (Fig. 1A). It was noticed that CD276, CD47 and HAVCR2 were also highly expressed in the GBM samples of TCGA and GEPIA database (Fig. 1B-D). Additional survival analysis of immune checkpoint gene revealed that CD276, TNFSF14, VTCN1, ID01, CD40, TNFSF18, TIGIT, CD274, CD70 and HAVCR2 could influence the prognosis of GBM patients (Fig. 1E-H and Figure S1). In summary, this study identified CD276 and HAVCR2 as the genes of interest.

\section{Correlation Analysis Between CD276, HAVCR2 And Macrophages}

Macrophages are important TAMS cells which play an important role in GBM, especially in the M2 macrophages. It was observed that primarily the CD276, HAVCR2 had higher expression in macrophages after clustering RNA single-cell sequencing data, whereas its expression in the neuronal cells was low (Fig. 2E-F and Figure S2), and associated with macrophage M2 infiltration (Figure S4). The analysis outcome also showed that the macrophage expression could affect the prognosis of GBM patients (Figure S3).

\section{GBM Poor Prognostic Immune Subtype Determination}


While a positive correlation was observed between CD276, HAVCR2 and CD163, no significant correlation was noticed with NOS2 (Fig. 3 and Figure S8A). By further re-grouping the data by the cut-off best truncation values, namely I (CD276 ${ }^{\text {low }} C D 163^{\text {low }} /$ HAVCR2 $\left.{ }^{\text {low }} C D 163^{\text {low }}\right)$, II (CD276 low

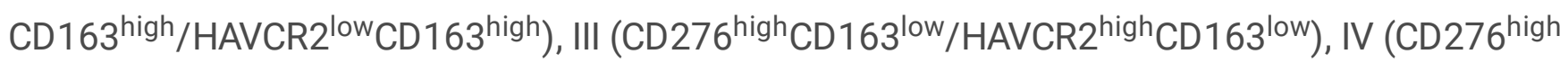
$\left.\mathrm{CD} 163^{\text {high }} / \mathrm{HAVCR} 2^{\text {high }} \mathrm{CD} 163^{\text {high }}\right)$, it was found that the IV group had the worst prognosis, I group had the best prognosis, and the results were statistically significant (Fig. 4). To verify this study's results, IHC staining was performed on paraffin sections of patients who had been in our hospital for the past five years and were pathologically diagnosed as high-grade glioma (Fig. 5A). It was noted that the degree of staining of paraffin sections was different in different patients undergoing the same treatment. The patients were also grouped as per the same best cut-off value after the quantification of the sections. The results showed that patients with IV group had the lowest survival whereas I group had the highest survival, which was also statistically significant (Fig. 5B and C). The four groups were associated with immune score and stromal score: I group had the lowest immune score and stromal score, while IV group had the highest immune score and stromal score (Figure S5), and macrophage infiltration in IV group was the highest (Figure S6).

\section{GSVA Enrichment Analysis}

Through the analysis of the GSE84465 data set, it was noted that the macrophages were divided into 0,2 clusters, wherein 0 cluster were basically tumor-associated macrophages and the 2 cluster were basically paracancerous cells. Furthermore, through the GSVA enrichment analysis and differential expression analysis, it was ascertained that there were differential signaling pathways (Fig. 6A). After further calculation gene set score, it was associated with EMT and JAK-STAT3 signaling pathway (Fig. 6B and C). GSVA analysis of CD276, HAVCR2 and CD163 showed that was positively correlated with macrophage activation, differentiation, EMT and JAK-STAT signaling pathway. This result can be verified in both TCGA and CGGA databases (Fig. 7).

High expression of CD276, HAVCR2 and CD163 was closely related to EMT and affected the prognosis of patients with high expression of PD-L1.

TAMS and EMT were closely related and enrichment analysis found significant enrichment in EMT signaling pathways, GSVA enrichment analysis of CD276, HAVCR2 and CD163 also found significant correlation with the EMT. Further analysis found that EMT most of the associated genes were significantly high expression in IV group (Fig. 8). Interestingly, IV group accounted for more than $30 \%$ of the patients in the PD-L1 high expression group, and IV group had a worse prognosis than the other three groups (Fig. 9). In order to further explore the reasons for the difference in prognosis, we establishing a risk model by EMT related genes, calculated the possible risk values for each patient (Figure S7), and found that IV group was also closely related to the high expression of EMT related genes in the PD-L1 high expression group, and were more likely to develop EMT high risk scores, and IV_EMT_score ${ }^{\text {high }}$ had a 
worse prognosis (Fig. 10). We speculated that this might be one of the reasons for the individualized differences in Anti-PDL1 treatment.

\section{Determination Of Upstream Transcription Factors}

We analyzed the differential expression of tumor-associated transcription factors $(n=318)$ downloaded from the CISTROME database using TCGA GBM data combined with GET data, the screening conditions were $|\mathrm{Log} F C|>1, \mathrm{P}<0.05$, and finally 58 differentially expressed transcription factors were obtained. We further analyzed the correlation with CD276/HAVCR2, the screening conditions were $|R|>0.5, P<0.05$. We found that the positive correlation between RUNX1/IKZF1 with CD276/HAVCR2 was the highest (Fig. 11A), and the correlation coefficient was $0.7,0.79$, respectively. Meanwhile, we predicted the transcription factors that might bind to CD276/HAVCR2 by UCSC database (Minimum score $=400$ ), and then cross with CD276/HAVCR2 related transcription factors, finally, the RUNX1 and IKZF1 was obtained (Fig. 11B and C). Further analysis found that The RUNX1/IKZF1 binding peaks were found in CD276/HAVCR2 promoter region by UCSC analysis (Fig. 11C and E). To search for specific possible binding sites, we screened the possible binding sites in the CD276/HAVCR2 sense sequence or antisense strand by JASPAR database, the screening conditions were Relative profile score threshold $>90 \%$, and finally the possible binding sites were tcctgcggttg, caaagaggaagc, respectively (Fig. 11D and F).

\section{Discussion}

Immune checkpoint inhibitors are yet to attain a clear clinical benefits in GBM [37]. Hence, an improved understanding of the possible causes of individual differences is needed to identify a subset of patients that may benefit from such treatments, thereby improving their current treatment. CD276 and HAVCR2 are key immune checkpoints in GBM, and its high expression is associated with poor prognosis in the patients $[34,35,38-40]$. This observation is consistent with the results of this study. Furthermore, CD163 is considered the most specific marker of TAMS, involved in the occurrence, development, migration, angiogenesis, EMT, etc., of GBM [41, 42]. PD-L1 is the focus of current immunotherapy research, the study found that its expression is closely related to EMT [43,44], and EMT is the focus of current cancer resistance research $[45,46]$. But no research has been done to explore the causes of individualization of patient therapy through their relationship. So, an important purpose of this study to find the additional factors that may affect the efficacy of immunosuppressant therapy and also consisted of a simple exploration of the mechanisms that may be involved.

Macrophages are important cellular components in tumor microenvironment. This study's cluster analysis of single-cell RNA sequencing data showed that CD276 and HAVCR2 was mainly highly expressed in macrophages and not in neuronal cells. Additionally, the TCGA and CGGA databases analysis showed that while CD276 and HAVCR2 had positive correlation with CD163, it had no significant correlation with NOS2. Results indicated that the existence of some close relationship between CD276, HAVCR2 and CD163. Hence, to further investigate the relationship between them, CD163, CD276 and 
HAVCR2 were re-grouped by the cut-off best truncation values. The outcomes showed that the patients with CD276 high/low expression also had CD163 high/low expression. The same phenomenon was exhibited in HAVCR2. The prognosis of CD163 high/low expression is different in patients with CD276/HAVCR2 high/low expression, and the prognosis of IV group is the worst, while that of I group is the best. This result was validated in our clinical data collection. The most widely studied immune checkpoint in the field of cancer is PD-L1/PD-1. But study found that the therapeutic effect was not satisfactory and there were differences in therapeutic effect [47]. Current clinical studies of non-small cell lung cancer have assumed that people with PD-L1 high levels of expression benefit more from the use of immune checkpoint inhibitors than patients with low or no PD-L1 expression [48]. Although there are still differences in individual treatments, this has prompted us to explore whether there is such a phenomenon in the GBM, thus enabling us to further individualize treatment. To further look for possible reasons, we subsequent analysis found that CD276, HAVCR2, CD163 and EMT might closely related, so we analyzed the EMT related genes and found a significant positive correlation with the IV group. Interestingly the high expression group of PD-L1 included more than $30 \%$ IV group patients, the prognosis of the IV group was significantly worse than that of the other three groups. The IV group of PD-L1 1 high expression was also significantly positively correlated with EMT related genes and patients were more likely to develop EMT high risk scores. This suggests that the EMT molecular subtypes in PDL1 1 high expression group were mainly the IV group, and IV_EMT_score ${ }^{\text {high }}$ had a worse prognosis. Therefore, the results showed that high expression of CD276, HAVCR2 and CD163 was closely related to EMT and affected the prognosis of patients with high expression of PD-L1, and might be one of the factors affecting the efficacy.

The possible involvement of them in biological processes and possible upstream mechanisms was also briefly explored in this study. The GSVA enrichment analysis and difference analysis of the GSE84465 data set highlighted the differences in several signaling pathways related to TAMS, including JAK-STAT, EMT and other signaling pathways. In addition, the CD276/HAVCR2 GSVA enrichment analysis also showed positive correlation with the positive regulation of EMT and JAK-STAT signaling pathway. This result can be verified in both TCGA and CGGA databases. Previous studies have shown a clear relationship between JAK-STAT signaling pathways, EMT and drug resistance [49], while TAMS can induce the occurrence of EMT[21], and studies in other tumors have found CD276,HAVCR2 and CD163 associated with AKT signaling pathways and EMT[50-52], but little is known about the relationship in GBM. To explore upstream regulatory factors that may affect CD276/HAVCR2 high expression in GBM. We analyzed tumor-associated transcription factors and found RUNX1/IKZF1 significantly positive correlation with CD276/HAVCR2, and corresponding binding peaks in the promoter regions of the CD276 sense sequence and the HAVCR2 antisense strand, indicating that there may be regulatory links between them. Previous studies have found that RUNX1/IKZF1 can induce the occurrence of EMT, thus affecting the occurrence and development of tumors [53-54]. But the relationship with CD276/HAVCR2 is not reported. we need further study to verify.

Nowadays, personalized treatment of patients is gaining in importance. Early detection of the key causes of individual differences in treatment of patients and the early formulation of personalized treatment can 
enable the patients to access to more treatment options. Though this study has reached the same conclusion through multi-database data analysis combined with the retrospective data that was collected in this study, the number of patients reviewed was small. Hence, more retrospective and prospective research support is required.

\section{Conclusion}

Despite the poor prognosis of GBM patients and the high postoperative recurrence rate and the use of immune checkpoint inhibitors provides additional treatment options for improving the prognosis of GBM patients. Through combined multi-database analysis, this study was able to identify immune subtypes with a good prognosis and factor that may affect the efficacy of Anti-PD-L1, thus providing a potential possibility for individualized treatment of immunity.

\section{Abbreviations}

CD276: CD276 Molecule; HAVCR2: Hepatitis A Virus Cellular Receptor 2; CD163: CD163 Molecule; RUNX1: RUNX Family Transcription Factor 1; IKZF1:IKAROS Family Zinc Finger1; GBM: Glioblastoma; EMT: Epithelial-mesenchymal transition; TAMS: Tumor-associated macrophages; TCGA: The Cancer Genome Atlas; CGGA: Chinese Glioma Genome Atlas; GEO: Gene Expression Omnibus; UCSC: the University of California Santa Cruz; GEPIA: Gene Expression Profiling Interactive Analysis; GO: Gene Ontology; KEGG: Kyoto Encyclopedia of Genes and Genomes; IHC: Immunohistochemistry; PD1: Anti-programmed death 1; PD-L1: Programmed death-ligand 1; FC: Fold change.

\section{Declarations}

\section{Acknowledgements}

The High-level Personnel Funded Projects, the Science and Technology benefits of Guizhou and the first people's hospital of Zunyi Joint research and development of science and technology projects (grant number[2010] 66, [2011] 24).

\section{Authors' contributions}

$\mathrm{XH}$ and SY designed and analyzed data; GZ and YF analyzed data; QZ and CX designed and supervised data analysis; $\mathrm{XH}$ and FC wrote the manuscript. All authors read and approved the fnal manuscript.

\section{Funding}

This research was funded by Natural Science Foundation of China (81660421).

\section{Availability of data and materials}


The data that support the findings of this study are openly available in TCGA at http://www.tcga.org/, CGGA at http://www.cgga.org.cn/, TIMER at https://cistrome.shinyapps.io/timer/, UCSC at https://xenabrowser.net/datapages/, GEO at https://www.ncbi.nlm.nih.gov/geo/ and MsigDB at https://www.gsea-msigdb.org/gsea/msigdb/.

\section{Ethics approval and consent to participate}

The studies involving human participants were reviewed and approved by the First Affiliated Hospital of Zunyi Medical University.

\section{Consent for publication}

Not applicable.

\section{Competing interests}

The authors declare that they have no competing interests.

\section{Author details}

1,2Department of Cerebrovascular Disease, the First Affiliated Hospital of Zunyi Medical University, Zunyi, Guizhou, 563000, China

\section{References}

1. Yun TJ, Park CK, Kim TM, Lee SH, Kim JH, Sohn CH, et al. Glioblastoma treated with concurrent radiation therapy and temozolomide chemotherapy: differentiation of true progression from pseudoprogression with quantitative dynamic contrast-enhanced MR imaging. Radiology. 2015;274:830-40.

2. Shenouda G, Souhami L, Petrecca K, Owen S, Panet-Raymond V, Guiot MC, et al. A Phase 2 Trial of Neoadjuvant Temozolomide Followed by Hypofractionated Accelerated Radiation Therapy With Concurrent and Adjuvant Temozolomide for Patients With Glioblastoma. Int J Radiat Oncol Biol Phys. 2017;97:487-94.

3. Norden AD, Korytowsky B, You M, Kim Le T, Dastani H, Bobiak S, et al. A Real-World Claims Analysis of Costs and Patterns of Care in Treated Patients with Glioblastoma Multiforme in the United States. Journal of managed care specialty pharmacy. 2019;25:428-36.

4. Linz U. Commentary on Effects of radiotherapy with concomitant and adjuvant temozolomide versus radiotherapy alone on survival in glioblastoma in a randomised phase III study: 5-year analysis of the EORTC-NCIC trial (Lancet Oncol. 2009;10:459-466). Cancer. 2010;116:1844-6.

5. Weller M, Cloughesy T, Perry JR, Wick W. Standards of care for treatment of recurrent glioblastomaare we there yet? Neurooncology. 2013;15:4-27. 
6. Rusthoven CG, Koshy M, Sher DJ, Ney DE, Gaspar LE, Jones BL, et al. Combined-Modality Therapy With Radiation and Chemotherapy for Elderly Patients With Glioblastoma in the Temozolomide Era: A National Cancer Database Analysis. JAMA neurology. 2016;73:821-8.

7. Kim YH, Kim T, Joo JD, Han JH, Kim YJ, Kim IA, et al. Survival benefit of levetiracetam in patients treated with concomitant chemoradiotherapy and adjuvant chemotherapy with temozolomide for glioblastoma multiforme. Cancer. 2015;121:2926-32.

8. Pardoll DM. The blockade of immune checkpoints in cancer immunotherapy. Nature reviews Cancer. 2012;12:252-64.

9. Fong B, Jin R, Wang X, Safaee M, Lisiero DN, Yang I, et al. Monitoring of regulatory T cell frequencies and expression of CTLA-4 on T cells, before and after DC vaccination, can predict survival in GBM patients. PloS one. 2012;7:e32614.

10. De Felice F, Musio D, Cassese R, Gravina GL, Tombolini V. New Approaches in Glioblastoma Multiforme: The Potential Role of Immune- check Point Inhibitors. Curr Cancer Drug Targets. 2017;17:282-9.

11. Caccese M, Indraccolo S, Zagonel V, Lombardi G. PD-1/PD-L1 immune-checkpoint inhibitors in glioblastoma: A concise review. Crit Rev Oncol Hematol. 2019;135:128-34.

12. Wang X, Guo G, Guan H, Yu Y, Lu J, Yu J. Challenges and potential of PD-1/PD-L1 checkpoint blockade immunotherapy for glioblastoma. Journal of experimental clinical cancer research: CR. 2019;38:87.

13. Saha D, Martuza RL, Rabkin SD. Macrophage Polarization Contributes to Glioblastoma Eradication by Combination Immunovirotherapy and Immune Checkpoint Blockade. Cancer cell. 2017;32:253 67.e5..

14. Heynckes S, Daka K, Franco P, Gaebelein A, Frenking JH, Doria-Medina R, et al. Crosslink between Temozolomide and PD-L1 immune-checkpoint inhibition in glioblastoma multiforme. BMC Cancer. 2019;19:117.

15. Cloughesy TF, Mochizuki AY, Orpilla JR, Hugo W, Lee AH, Davidson TB, et al. Neoadjuvant anti-PD-1 immunotherapy promotes a survival benefit with intratumoral and systemic immune responses in recurrent glioblastoma. Nature medicine. 2019;25:477-86.

16. Zeng J, See AP, Phallen J, Jackson CM, Belcaid Z, Ruzevick J, et al. Anti-PD-1 blockade and stereotactic radiation produce long-term survival in mice with intracranial gliomas. Int $\mathrm{J}$ Radiat Oncol Biol Phys. 2013;86:343-9.

17. Kim JE, Patel MA, Mangraviti A, Kim ES, Theodros D, Velarde E, et al. Combination Therapy with AntiPD-1, Anti-TIM-3, and Focal Radiation Results in Regression of Murine Gliomas. Clinical cancer research: an official journal of the American Association for Cancer Research. 2017;23:124-36.

18. Sarcar B, Kahali S, Prabhu AH, Shumway SD, Xu Y, Demuth T, et al. Targeting radiation-induced G(2) checkpoint activation with the Wee-1 inhibitor MK-1775 in glioblastoma cell lines. Mol Cancer Ther. 2011;10:2405-14. 
19. Kesarwani P, Prabhu A, Kant S, Kumar P, Graham SF, Buelow KL, et al. Tryptophan Metabolism Contributes to Radiation-Induced Immune Checkpoint Reactivation in Glioblastoma. Clinical cancer research: an official journal of the American Association for Cancer Research. 2018;24:3632-43.

20. Seo JS, Lee JW, Kim A, Shin JY, Jung YJ, Lee SB, et al. Whole Exome and Transcriptome Analyses Integrated with Microenvironmental Immune Signatures of Lung Squamous Cell Carcinoma. Cancer immunology research. 2018;6:848-59.

21. Han Y, Guo W, Ren T, Huang Y, Wang S, Liu K, et al. Tumor-associated macrophages promote lung metastasis and induce epithelial-mesenchymal transition in osteosarcoma by activating the COX2/STAT3 axis. Cancer letters. 2019:116-25.

22. Wei C, Yang C, Wang S, Shi D, Zhang C, Lin X, et al. Crosstalk between cancer cells and tumor associated macrophages is required for mesenchymal circulating tumor cell-mediated colorectal cancer metastasis. Mol Cancer. 2019;18:64.

23. Garris C, Pittet MJ. Therapeutically reeducating macrophages to treat GBM. Nature medicine. 2013;19:1207-8.

24. Yang L, Zhang Y. Tumor-associated macrophages: from basic research to clinical application. J Hematol Oncol. 2017;10:58.

25. Grivennikov SI, Greten FR, Karin M. Immunity, inflammation, and cancer. Cell. 2010;140:883-99.

26. Van Gorp H, Delputte PL, Nauwynck HJ. Scavenger receptor CD163, a Jack-of-all-trades and potential target for cell-directed therapy. Molecular immunology. 2010;47:1650-60.

27. Franco R, Fernández-Suárez D. Alternatively activated microglia and macrophages in the central nervous system. Progress in neurobiology. 2015;131:65-86.

28. Colin S, Chinetti-Gbaguidi G, Staels B. Macrophage phenotypes in atherosclerosis. Immunological reviews. 2014;262:153-66.

29. Chen T, Chen J, Zhu Y, Li Y, Wang Y, Chen H, et al. CD163, a novel therapeutic target, regulates the proliferation and stemness of glioma cells via casein kinase 2. Oncogene. 2019;38:1183-99.

30. Liu S, Zhang C, Maimela NR, Yang L, Zhang Z, Ping Y, et al. Molecular and clinical characterization of CD163 expression via large-scale analysis in glioma. Oncoimmunology. 2019;8:1601478.

31. Hu Y, He MY, Zhu LF, Yang CC, Zhou ML, Wang Q, et al. Tumor-associated macrophages correlate with the clinicopathological features and poor outcomes via inducing epithelial to mesenchymal transition in oral squamous cell carcinoma. Journal of experimental clinical cancer research: CR. 2016;35:12.

32. Koelzer VH, Canonica K, Dawson H, Sokol L, Karamitopoulou-Diamantis E, Lugli A, et al. Phenotyping of tumor-associated macrophages in colorectal cancer: Impact on single cell invasion (tumor budding) and clinicopathological outcome. Oncoimmunology. 2016;5:e1106677.

33. Kraan J, van den Broek P, Verhoef C, Grunhagen DJ, Taal W, Gratama JW, et al. Endothelial CD276 (B7-H3) expression is increased in human malignancies and distinguishes between normal and tumour-derived circulating endothelial cells. British journal of cancer. 2014;111:149-56. 
34. Takashima Y, Kawaguchi A, Hayano A, Yamanaka R. CD276 and the gene signature composed of GATA3 and LGALS3 enable prognosis prediction of glioblastoma multiforme. PloS one. 2019;14:e0216825.

35. Hung AL, Garzon-Muvdi T, Lim M. Biomarkers and Immunotherapeutic Targets in Glioblastoma. World neurosurgery. 2017;102:494-506.

36. Anderson AC, Anderson DE, Bregoli L, Hastings WD, Kassam N, Lei C, et al. Promotion of tissue inflammation by the immune receptor Tim-3 expressed on innate immune cells. 318. New York: Science; 2007. pp. 1141-3.

37. Xue Y, Tong L, LiuAnwei Liu F, Liu A, Zeng S, Xiong Q, et al. Tumorinfiltrating M2 macrophages driven by specific genomic alterations are associated with prognosis in bladder cancer. Oncol Rep. 2019;42:581-94.

38. Zhang J, Wang J, Marzese DM, Wang X, Yang Z, Li C, et al. B7H3 regulates differentiation and serves as a potential biomarker and theranostic target for human glioblastoma. Lab Invest. 2019;99:111729.

39. Wang Z, Wang Z, Zhang C, Liu X, Li G, Liu S, et al. Genetic and clinical characterization of B7-H3 (CD276) expression and epigenetic regulation in diffuse brain glioma. Cancer Sci. 2018;109:2697705.

40. Li G, Wang Z, Zhang C, Liu X, Cai J, Wang Z, et al. Molecular and clinical characterization of TIM-3 in glioma through 1,024 samples. Oncoimmunology. 2017;6:e1328339.

41. Zhang M, Hutter G, Kahn SA, Azad TD, Gholamin S, Xu CY, et al. Anti-CD47 Treatment Stimulates Phagocytosis of Glioblastoma by M1 and M2 Polarized Macrophages and Promotes M1 Polarized Macrophages In Vivo. PloS one. 2016;11:e0153550.

42. Zhou W, Ke SQ, Huang Z, Flavahan W, Fang X, Paul J, et al. Periostin secreted by glioblastoma stem cells recruits M2 tumour-associated macrophages and promotes malignant growth. Nat Cell Biol. 2015;17:170-82.

43. Li F, Zhu T, Yue Y, Zhu X, Wang J, Liang L. Preliminary mechanisms of regulating PDL1 expression in nonsmall cell lung cancer during the EMT process. Oncol Rep. 2018;40:775-82.

44. Fei Z, Deng Z, Zhou L, Li K, Xia X, Xie R. PD-L1 Induces Epithelial-Mesenchymal Transition in Nasopharyngeal Carcinoma Cells Through Activation of the PI3K/AKT Pathway. Oncology research. 2019;27:801-7.

45. Shen M, Xu Z, Xu W, Jiang K, Zhang F, Ding Q, et al. Inhibition of ATM reverses EMT and decreases metastatic potential of cisplatin-resistant lung cancer cells through JAK/STAT3/PD-L1 pathway. Journal of experimental clinical cancer research: CR. 2019;38:149.

46. Du B, Shim JS. Targeting Epithelial-Mesenchymal Transition (EMT) to Overcome Drug Resistance in Cancer. Molecules (Basel, Switzerland). 2016;21.

47. de Groot J, Penas-Prado M, Alfaro-Munoz K, Hunter K, Pei BL, O'Brien B, et al. Window-of-opportunity clinical trial of pembrolizumab in patients with recurrent glioblastoma reveals predominance of immune-suppressive macrophages. Neurooncology. 2020;22:539-49. 
48. Ito K, Miura S, Sakaguchi T, Murotani K, Horita N, Akamatsu H, et al. The impact of high PD-L1 expression on the surrogate endpoints and clinical outcomes of anti-PD-1/PD-L1 antibodies in nonsmall cell lung cancer. Lung cancer (Amsterdam Netherlands). 2019;128:113-9.

49. Jin W. Role of JAK/STAT3 Signaling in the Regulation of Metastasis, the Transition of Cancer Stem Cells, and Chemoresistance of Cancer by Epithelial-Mesenchymal Transition. Cells. 2020;9.

50. Jiang B, Zhang T, Liu F, Sun Z, Shi H, Hua D, et al. The co-stimulatory molecule B7-H3 promotes the epithelial-mesenchymal transition in colorectal cancer. Oncotarget. 2016;7:31755-71.

51. Shan B, Man H, Liu J, Wang L, Zhu T, Ma M, et al. TIM-3 promotes the metastasis of esophageal squamous cell carcinoma by targeting epithelial-mesenchymal transition via the Akt/GSK-3 $\beta /$ Snail signaling pathway. Oncol Rep. 2016;36:1551-61.

52. Wei C, Yang C, Wang S, Shi D, Zhang C, Lin X, et al. M2 macrophages confer resistance to 5fluorouracil in colorectal cancer through the activation of CCL22/PI3K/AKT signaling. OncoTargets therapy. 2019;12:3051-63.

53. Fritz AJ, Hong D, Boyd J, Kost J, Finstaad KH, Fitzgerald MP, et al. RUNX1 and RUNX2 transcription factors function in opposing roles to regulate breast cancer stem cells. Journal of cellular physiology. 2020.

54. Hung JJ, Kao YS, Huang CH, Hsu WH. Overexpression of Aiolos promotes epithelial-mesenchymal transition and cancer stem cell-like properties in lung cancer cells. Scientific reports. 2019;9:2991.

\section{Supplementary Figure Legends}

Figure S1. Prognostic impact of other immune checkpoint genes in GBM patients, $\mathrm{P}<0.05$ of which was considered statistically significant.

Figure S2. CD276 and HAVCR2 expression in the GSE103224 dataset, they are mainly highly expressed in macrophages.

Figure S3. Effect of immune cells on patient prognosis. the figure shows that macrophages and B cells can affect the prognosis of patients, and CD8 and CD 4 cells have no significant effect on the prognosis of patients.

Figure S4. Relationship between CD276/HAVCR2 expression and immune cells infiltration in TIMER database.

Figure S5. Relationship between four subtypes and immune and stromal scores. (A-D) IV group had the highest immune score and stromal score, and I group had the lowest immune score and stromal score. *囚 $0.05, * * \otimes 0.01, * * * \otimes 0.001, * \star * \star \otimes 0.0001$.

Figure S6. The infiltration analysis of I-IV and immune cells showed that IV group had the largest

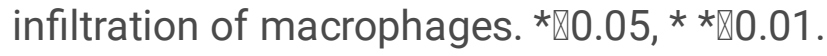


Figure S7. Lasso risk model construction by EMT related genes. $(A, B)$ The process of using Prognosis related genes to building the signature. (C,D) Kaplan-Meier overall survival of high/low-risk patients in TCGA and CGGA databases. (E,F) The ROC curves of the risk signature in TCGA and CGGA datasets.

\section{Figures}

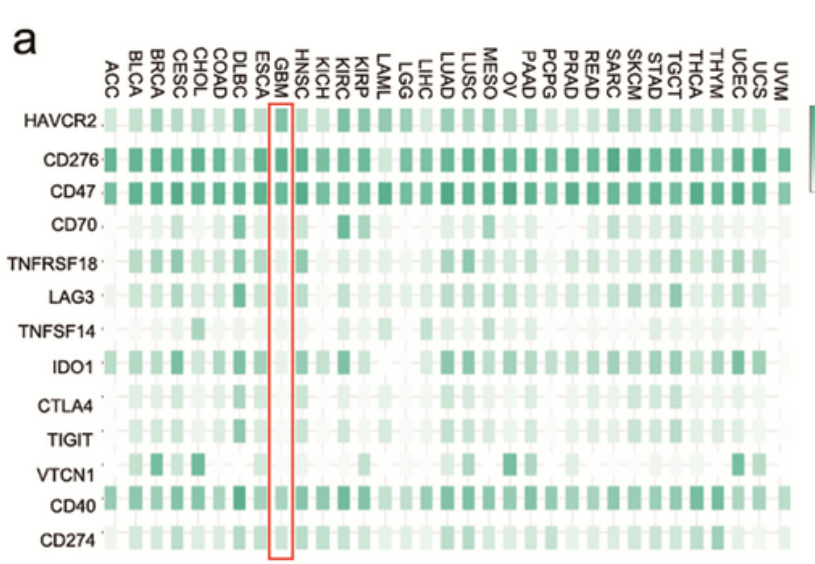

C
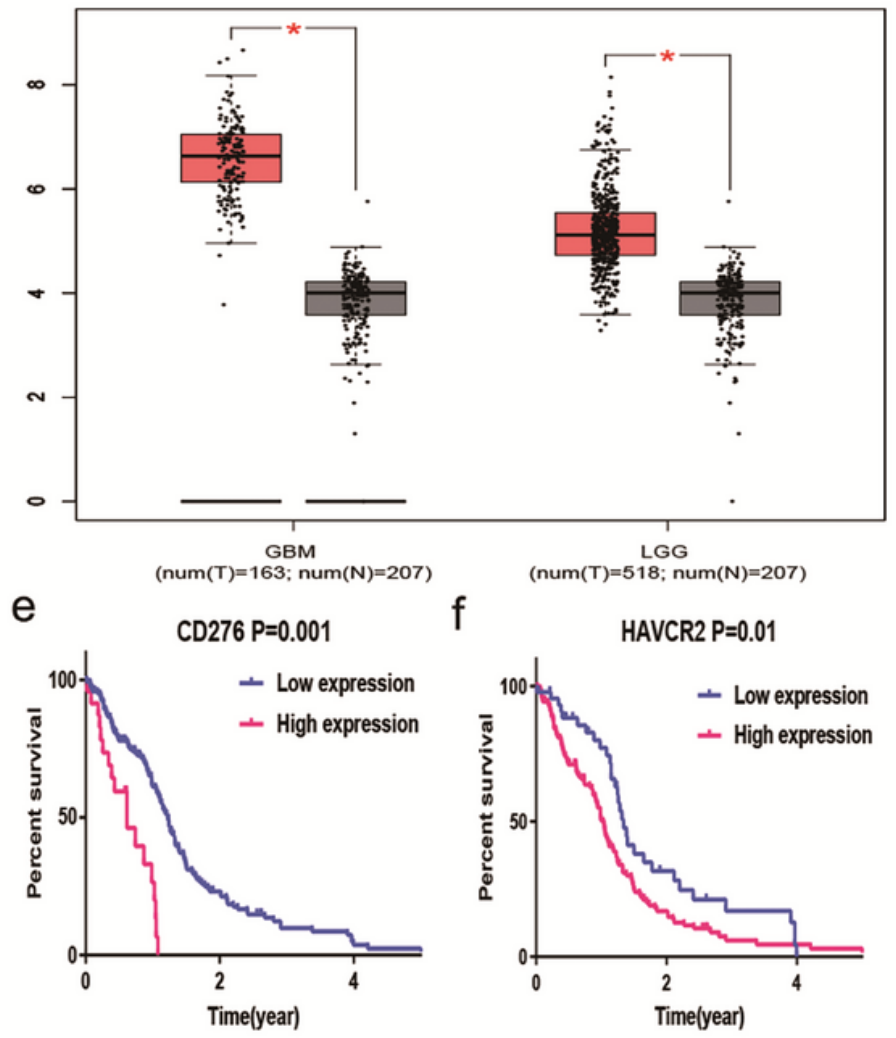

b

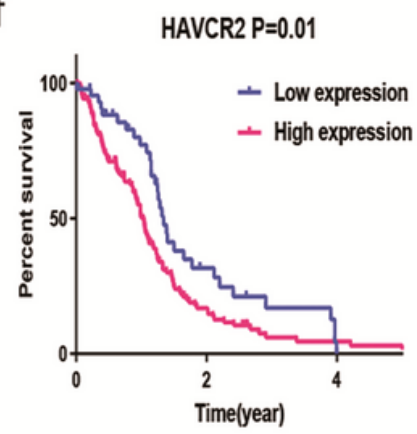

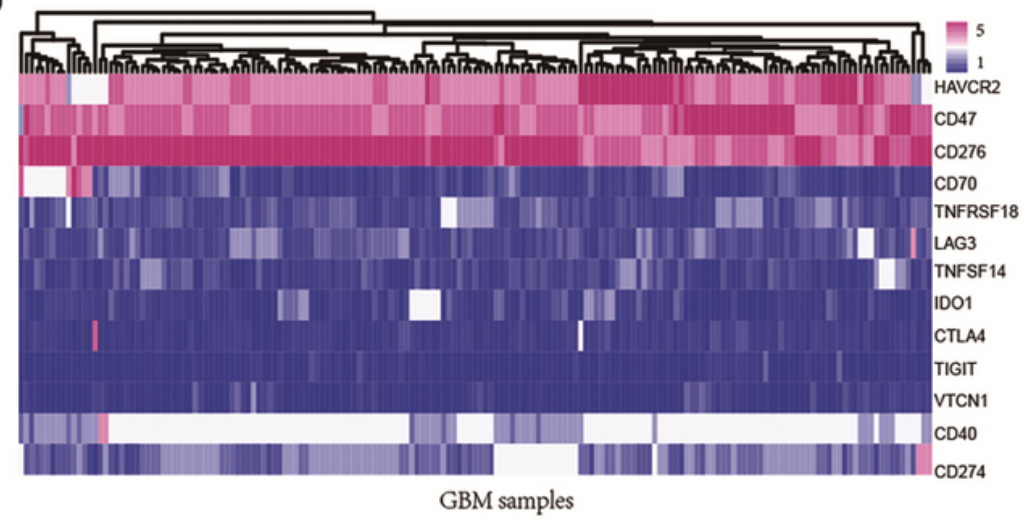

d

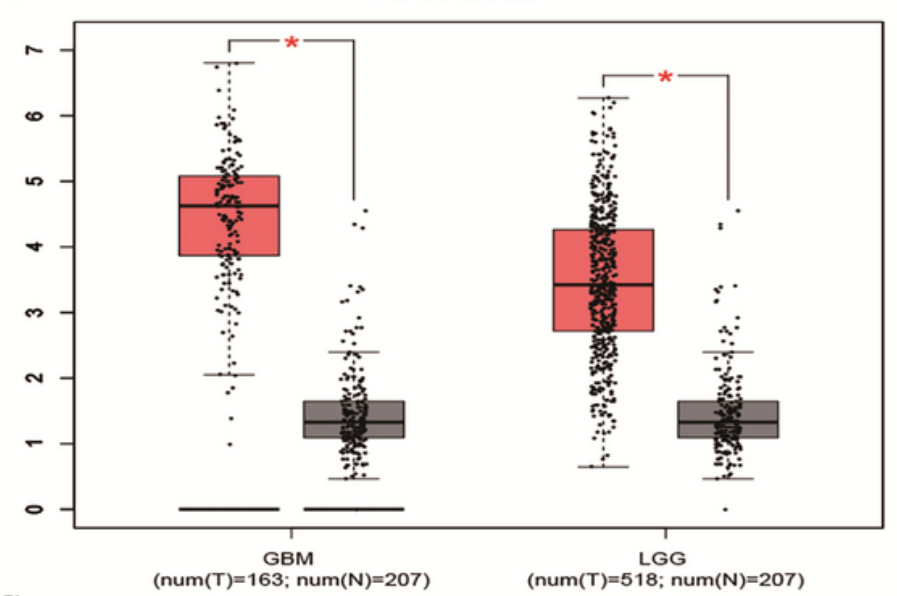

g

h

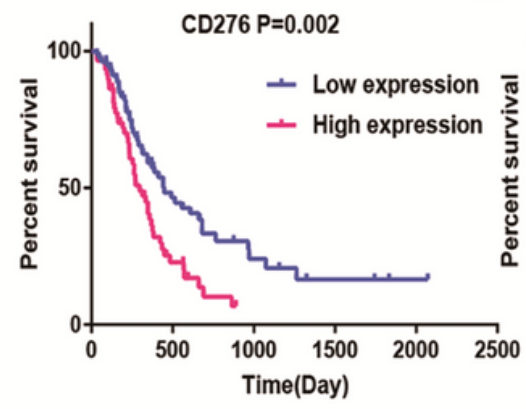

HAVCR2 $P=0.01$

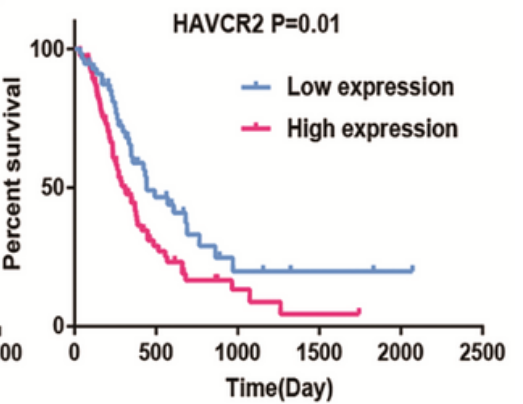

Figure 1

CD276 and HAVCR2 expression and prognosis in tumors. (A) Expression of immune checkpoint genes in 33 types of tumors, CD276 and HAVCR2 significantly higher in most tumors, including GBM. (B) Significant high expression of CD276 and HAVCR2 in GBM samples of TCGA databases. (C and D) Expression of CD276 and HAVCR2 in glioma and paracancerous tumors. Significantly high expression in 
lower grade glioma and GBM, and the difference was statistically significant. (E and F) CD276 and HAVCR2 high/low expression in the TCGA and CGGA database could affect the prognosis of GBM and poor prognosis of patients with high expression.

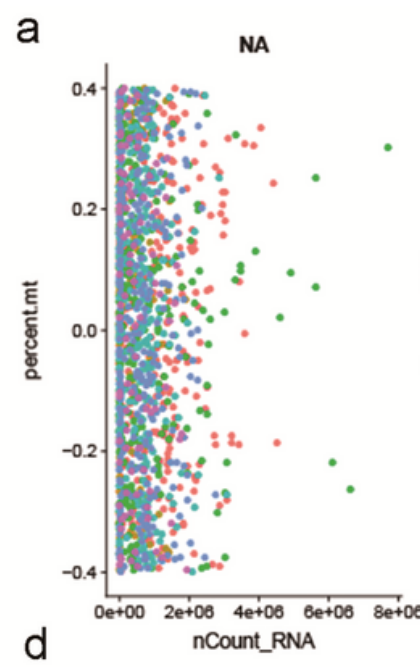

d

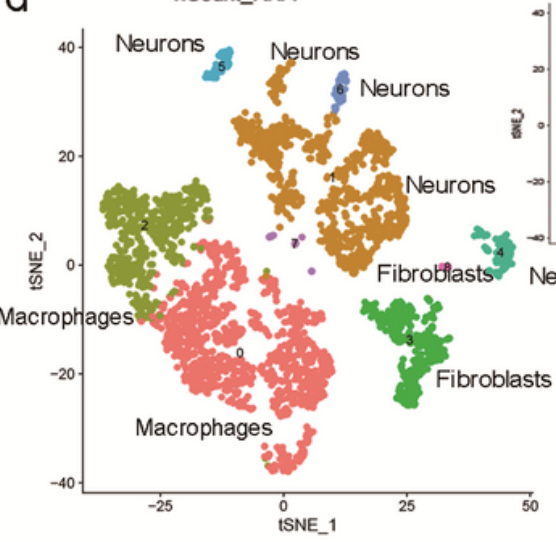

0.63

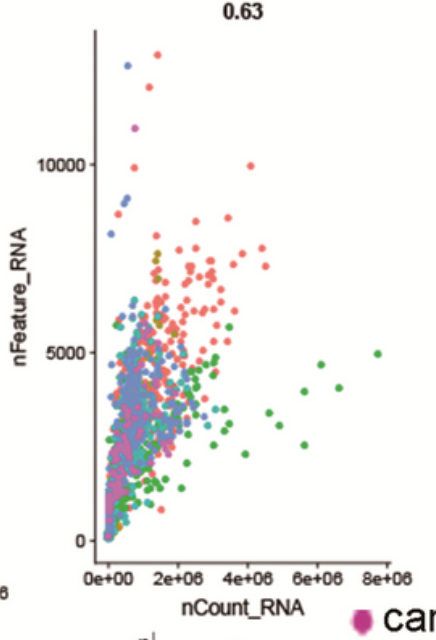

b

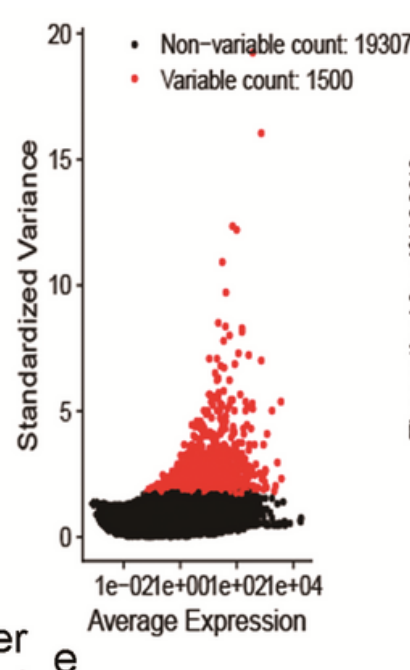

CD276

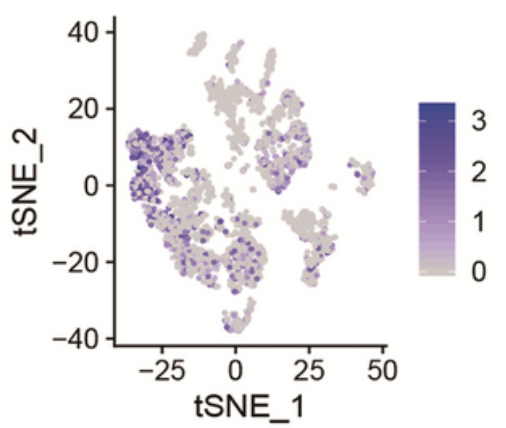

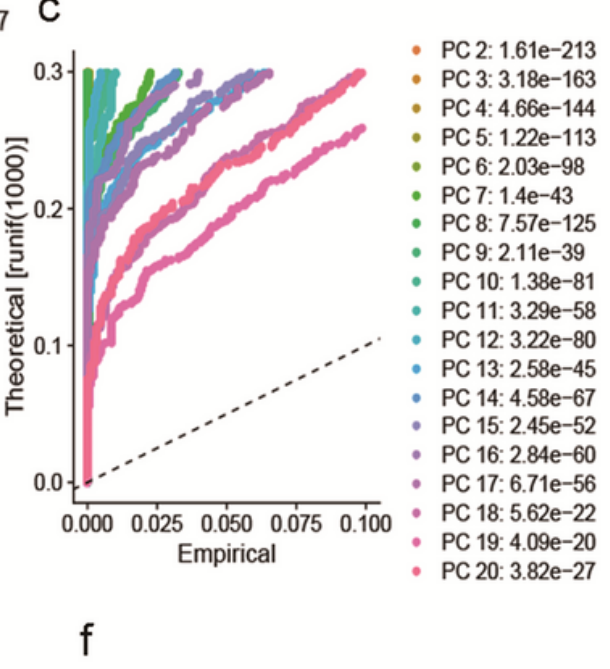

HAVCR2

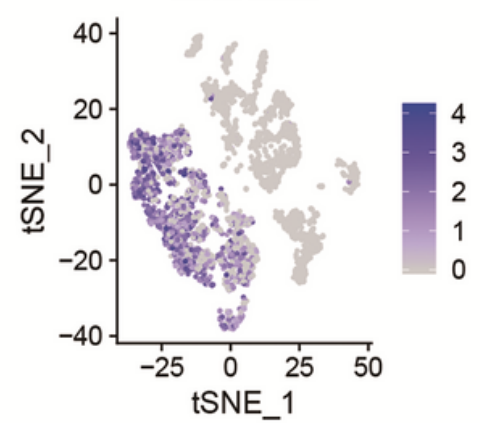

\section{Figure 2}

We show the sequencing analysis and quality control process RNA single cells by GSE84465 data sets. (A) We filter cells of poor quality, and no mitochondria were found in the single cell RNA sequencing data.

(B) We plot the characteristic variance map based on genes with significant differences. (C) After we analyzed the data principal components, the actual cell types were clustered using the effective PCA components and $P$ values. (D) We clustered according to different cell types, in figure 0,2 clusters are macrophages. (E-F) CD276 and HAVCR2 expression in each cluster of cells, the results showed significantly high expression in macrophages. 
a

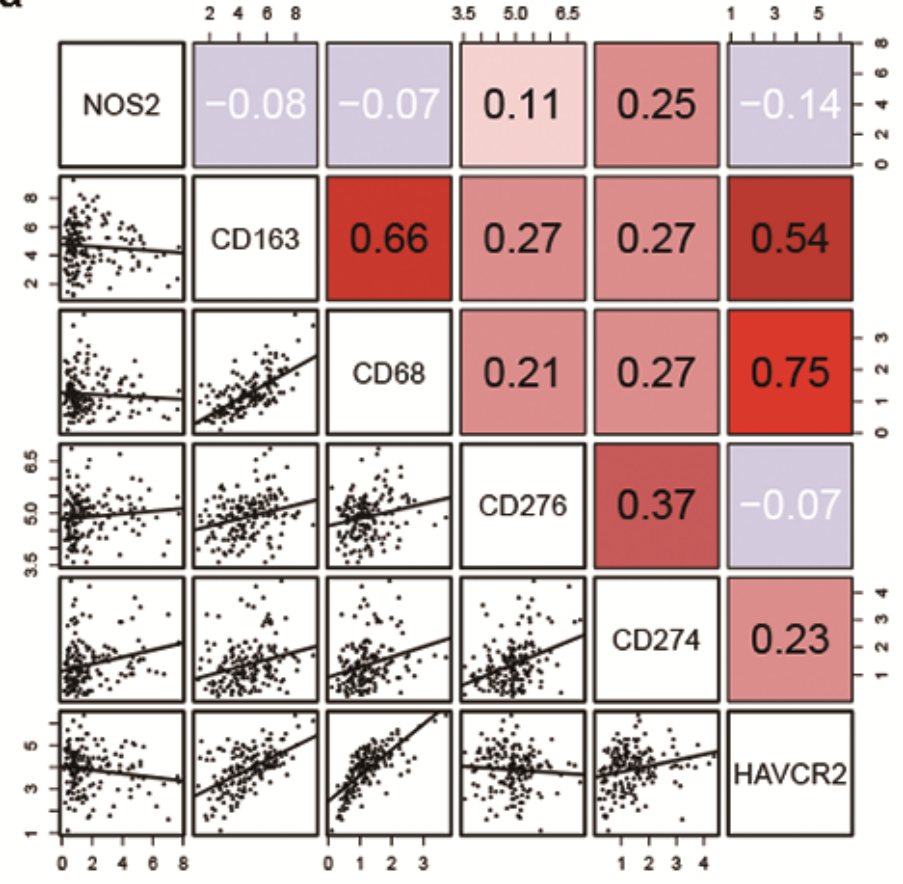

b

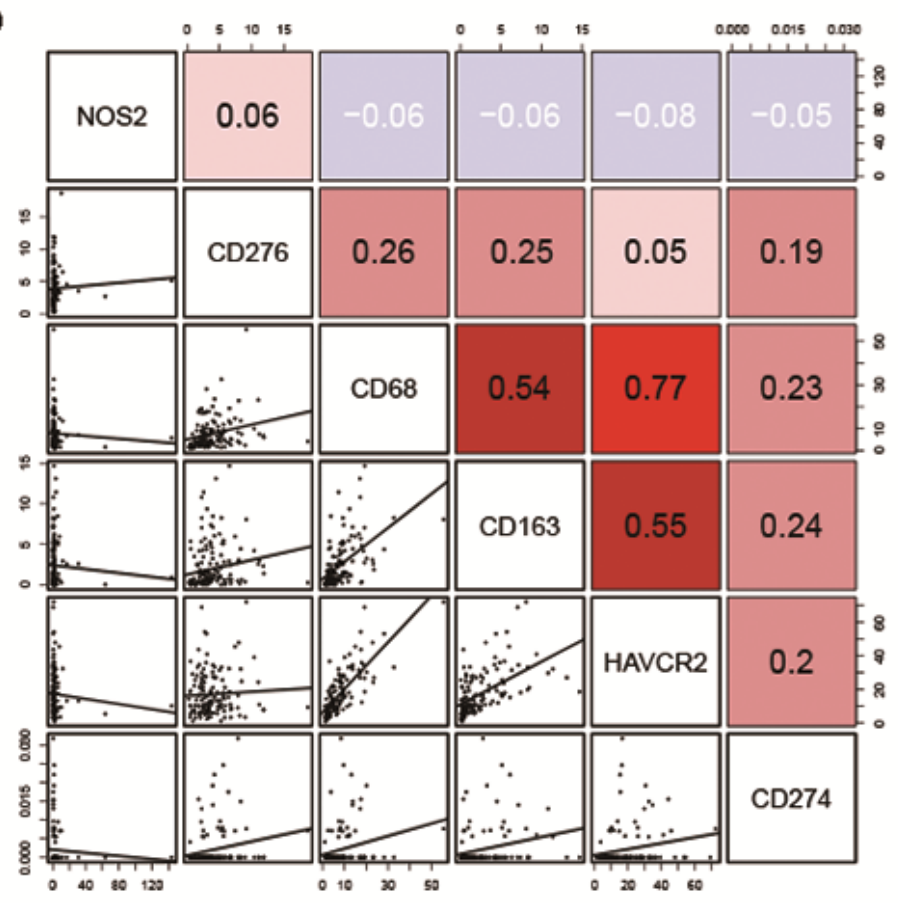

Figure 3

CD276, HAVCR2 and CD163 correlation analysis. (A and B)Their correlation analysis in the TCGA and CGGA databases showed a positive correlation with the CD163 and no significant correlation with the NOS2.
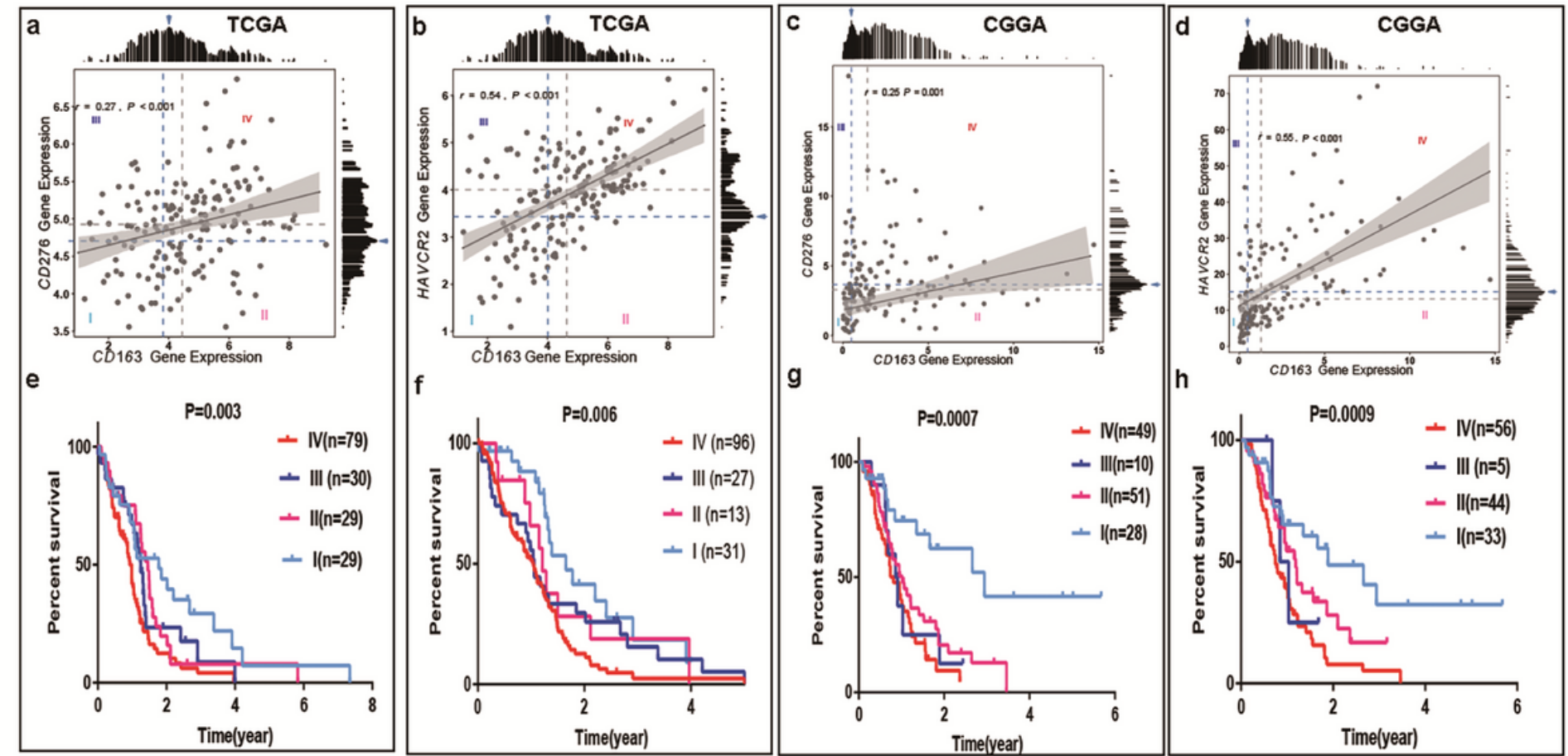

Figure 4 
(A-D) Scatter plots of CD276/HAVCR2 and CD163 in TCGA and CGGA databases, respectively, a linear regression line is plotted with the gray shaded region showing the $95 \%$ confidence interval. Pearson's correlation coefficient $r$ and $P$ values are given at the top. The median of CD276/HAVCR2 and cd163 expression is indicated by a gray dotted line. Log-rank statistics were applied to identify the optimal cutoff for transforming the continuous variable of gene expression into categorical high- and low-expression groups. The test score at each candidate cut-off across the log-transformed gene expression values was plotted. The highest value (indicated with a blue arrow) was using solid blue lines to best separating patients into 4 groups (named groups I to IV). (E-H) Represents the survival curves of I to IV groups in TCGA and CGGA databases, respectively. The prognosis was the best in group I and the worst in group IV (pष0.05).

a
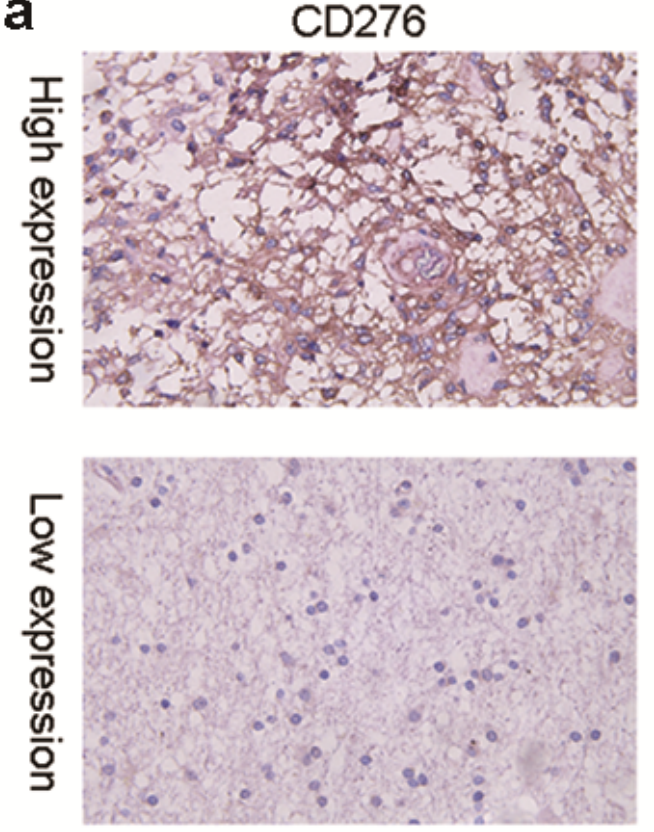

b

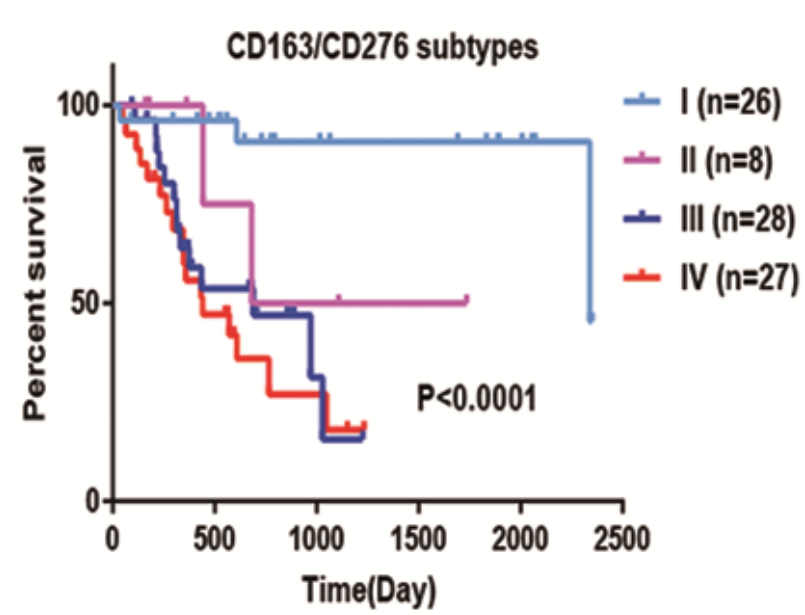

HAVCR2
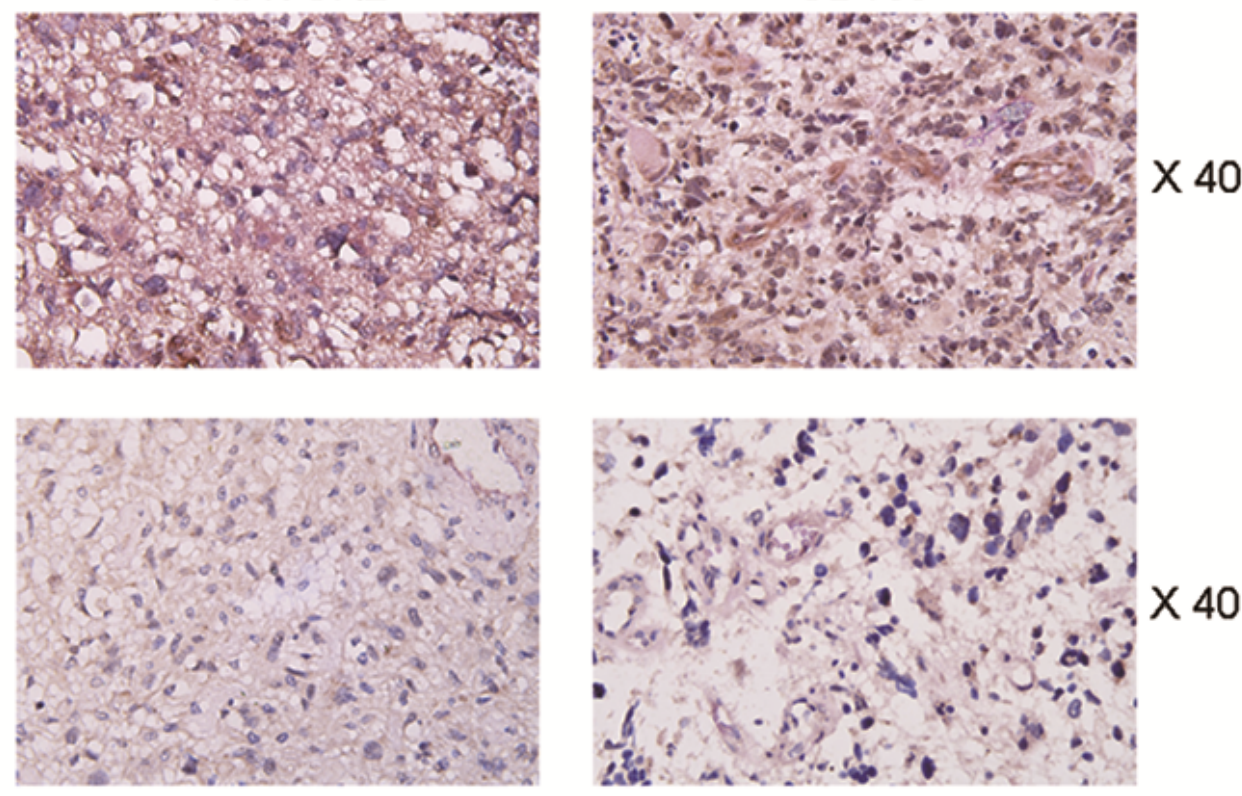

C

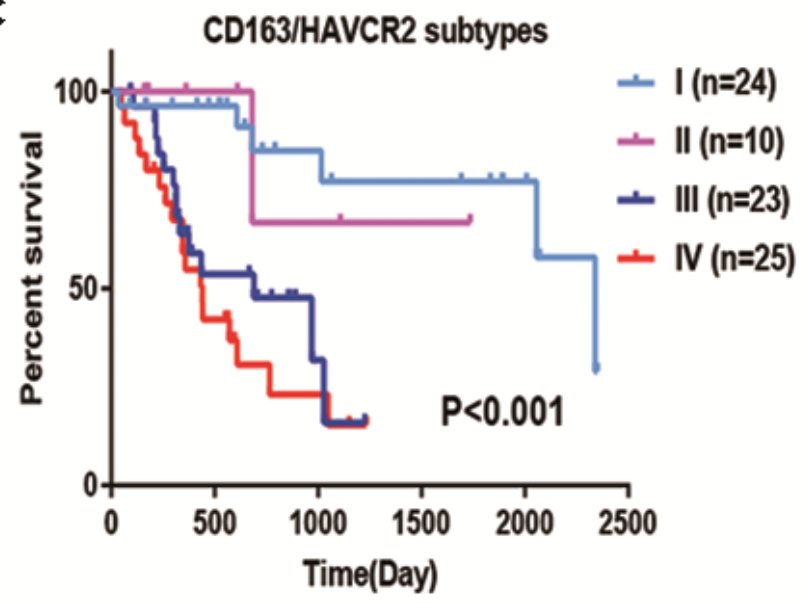

Figure 5

(A) ICH staining of CD276, HAVCR2 and CD163. (B and C) The best cut-off truncation value after ICH quantification was divided into four groups, and the survival curve was drawn according to the survival 
time and survival state of the patients. The results showed that IV group had the worst prognosis and I group had the best prognosis.

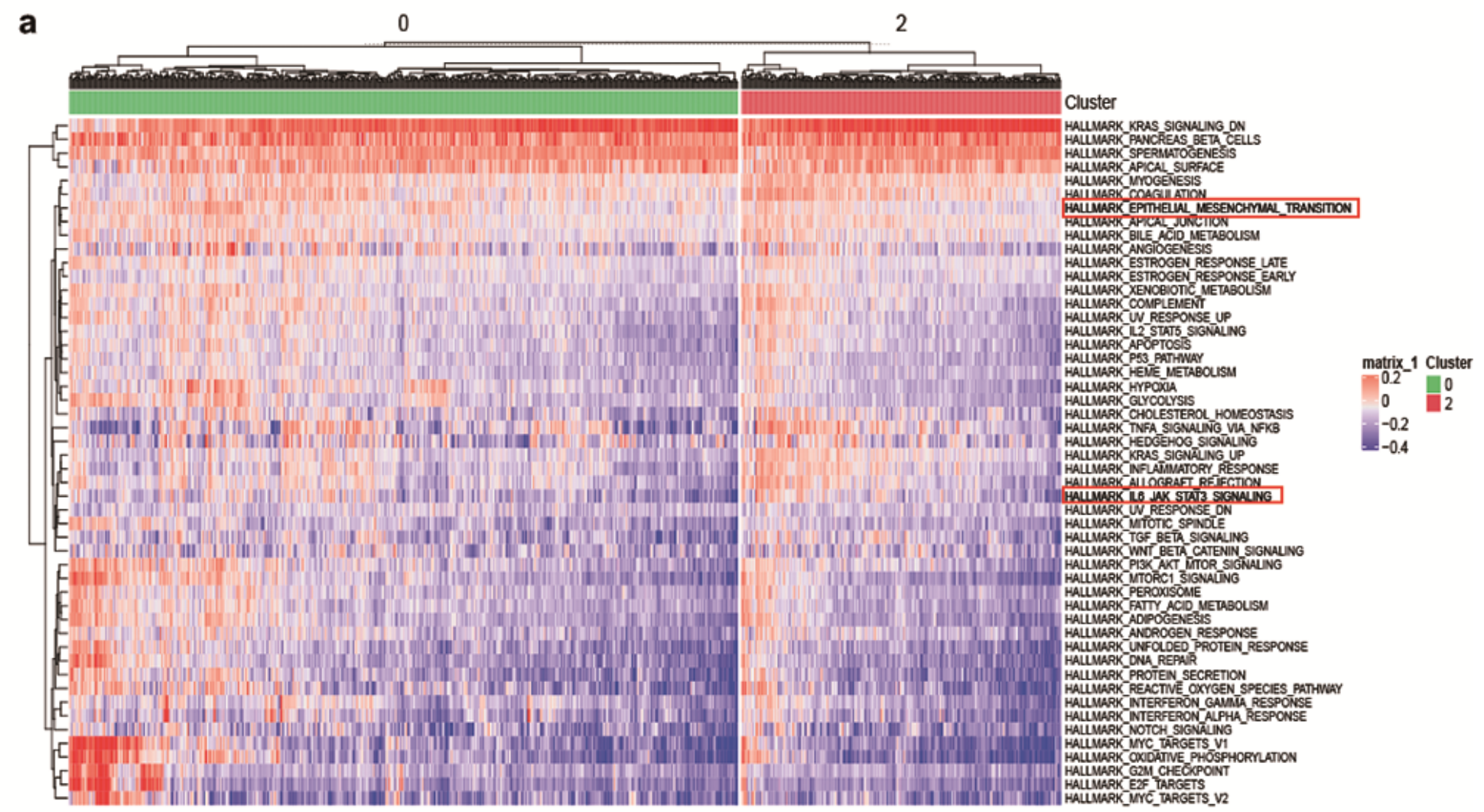

b

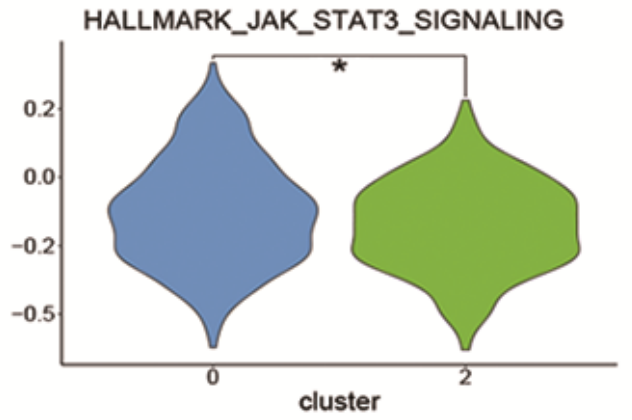

C HALLMARK_EPITHELIAL_MESENCHYMAL_TRANSITION

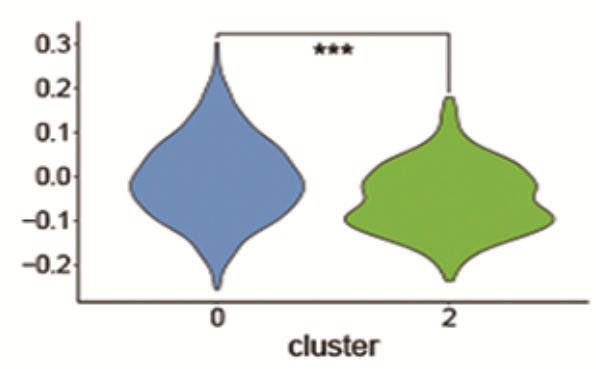

Figure 6

(A) The GSVA enrichment analysis and pathway difference analysis of 0 and 2 clusters after single-cell RNA sequencing analysis of GSE84465 data set showed that there were different signaling pathways. (B

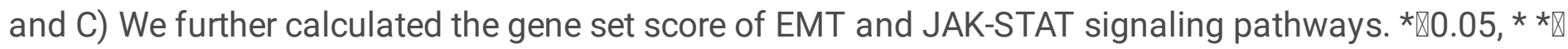
$0.01, * * * \mathbb{0} 0.001$. 

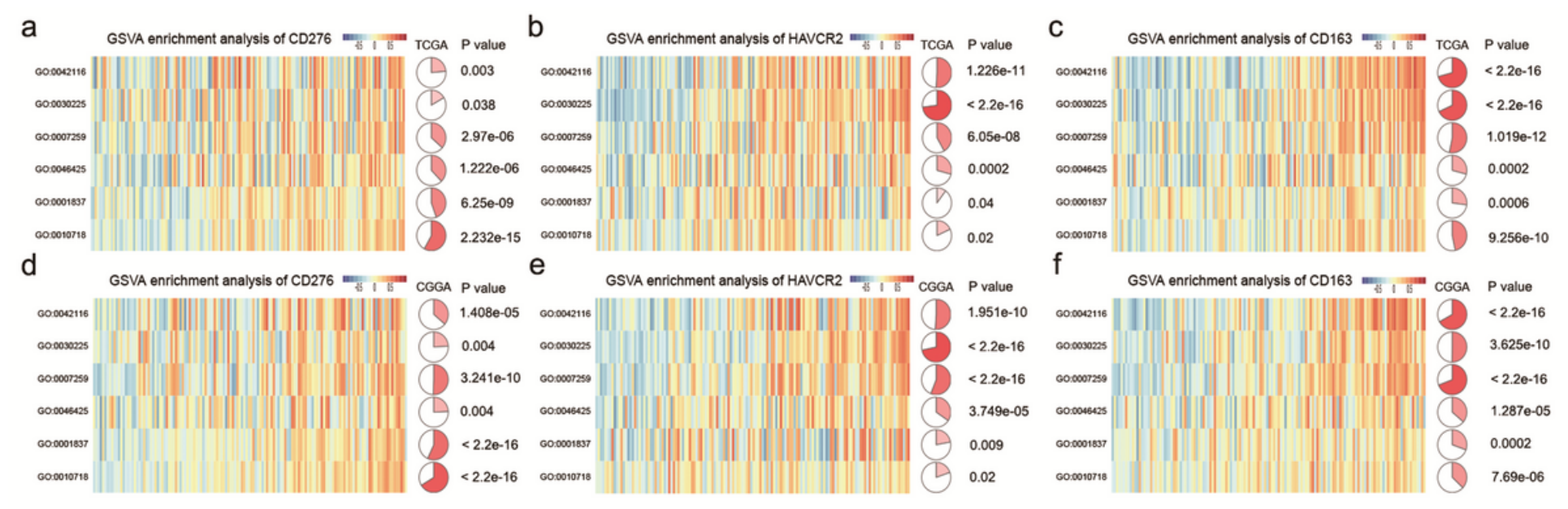

\section{Figure 7}

CD276, HAVCR2 and CD163 GSVA enrichment analysis. The red sector diagram indicates positive correlation, and the green sector diagram indicates negative correlation. The larger the correlation, the larger the area and the darker the color, and the results showed positive correlation with macrophage activation, differentiation, positive regulation of EMT and JAK-STAT regulation. $\mathrm{P}<0.05$ was considered statistically significant. GO: 0042116 macrophage activation, GO: 0030225 macrophage differentiation, GO: 0007259 receptor signaling pathway via JAK-STAT, GO: 0046425 regulation of receptor signaling pathway via JAK-STAT, GO: 0046427 positive regulation of receptor signaling pathway via JAK-STAT, GO:0001837 epithelial to mesenchymal transition, G0:0010718 positive regulation of epithelial to mesenchymal transition.
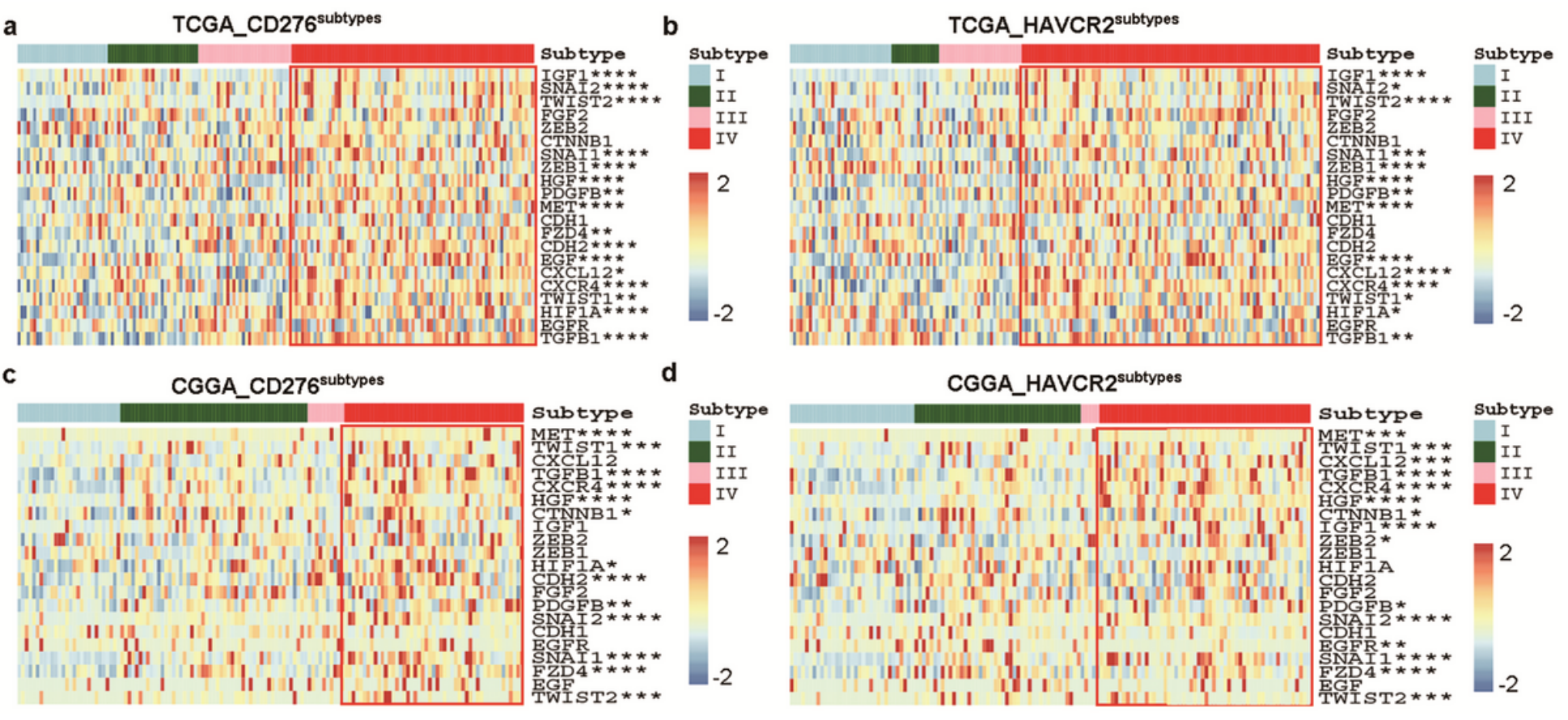

\section{Figure 8}


Correlation analysis of I-IV group and EMT related genes. Red indicates positive correlation and blue indicates negative correlation in heatmap. The results showed positive correlation between IV group and EMT gene expression. $* \llbracket 0.05, * * \llbracket 0.01, * * * \llbracket 0.001, * \star * * \llbracket 0.0001$.

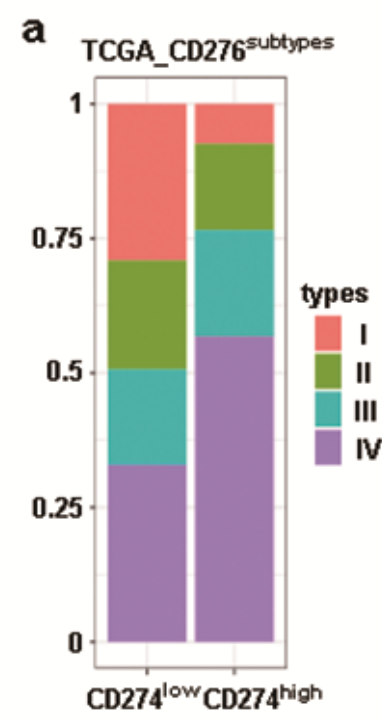

C TCGA_CD276 $6^{\text {suthpes }}$

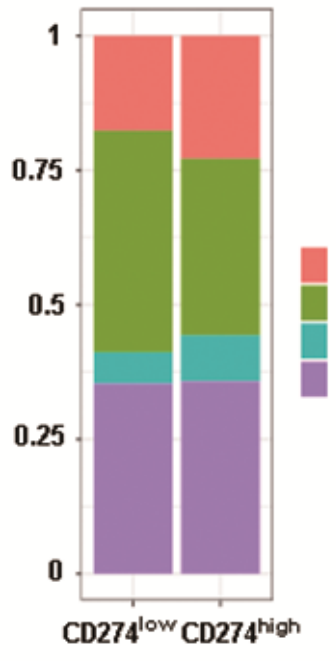

b

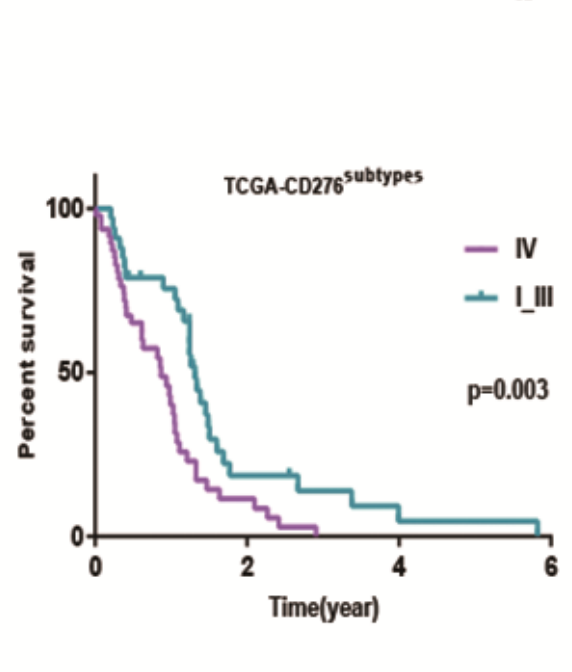

d

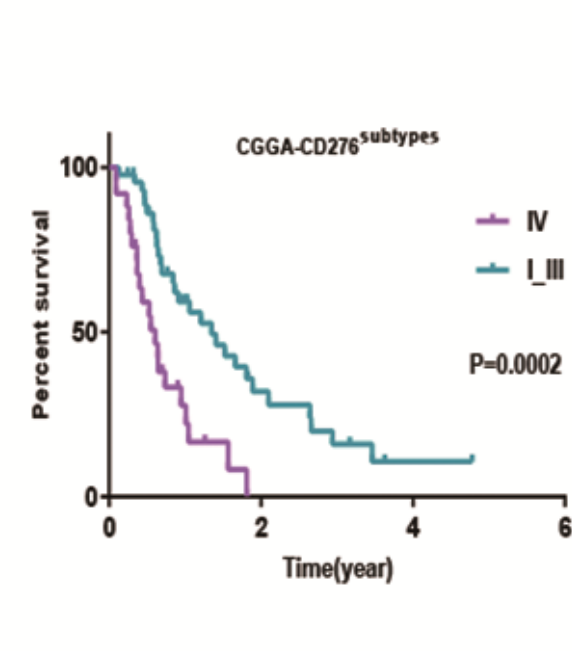

TCGA_HAVCR2 ${ }^{\text {subtypes }}$

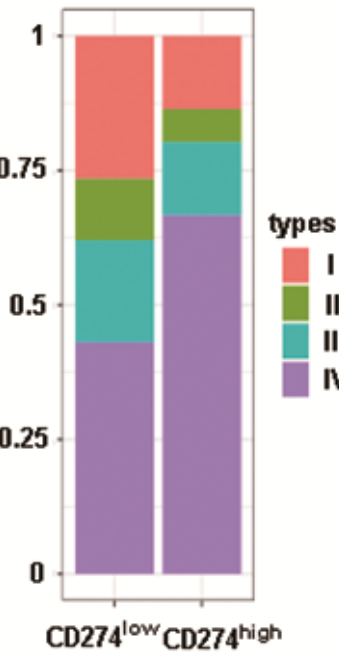

TCGA_HAVCR2 $2^{\text {suttppes }}$

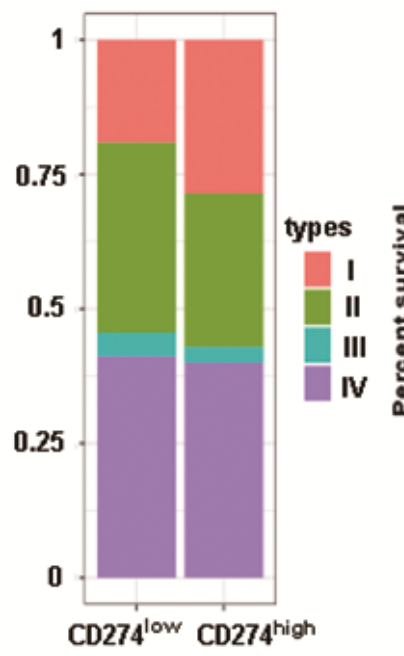

- IV

- IIIII

$P=0.09$

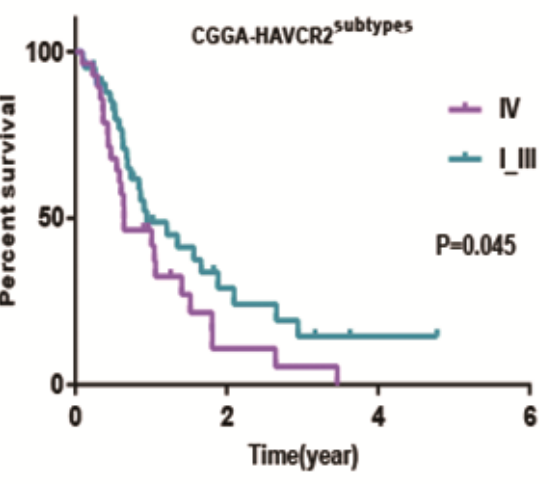

\section{Figure 9}

IV group accounted for more than $30 \%$ of the PD-L1 high expression group, and the prognosis of IV group was significantly worse than that of the other three groups, results are verified in the CGGA database. 

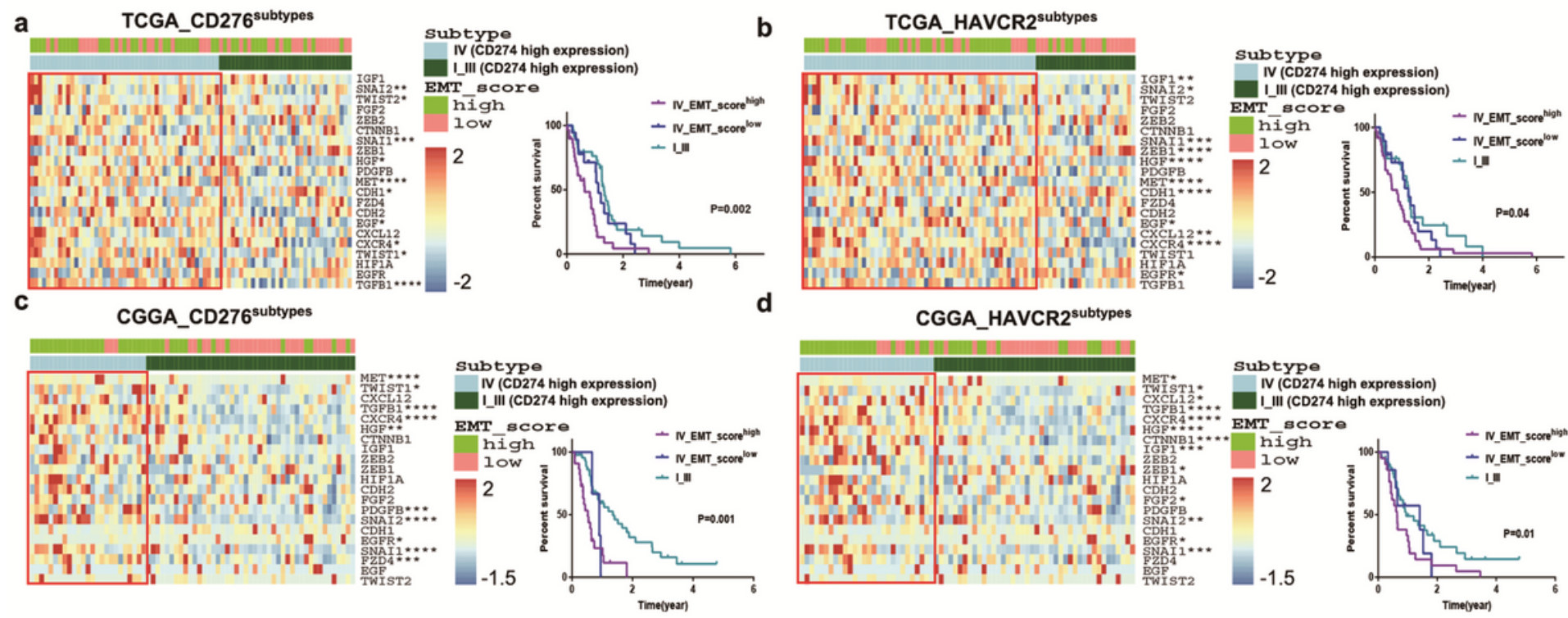

Figure 10

We found that IV group of patients were more likely to have high EMT scores, it was also closely related to the high expression of EMT related genes in the PD-L1 high expression group, and IV_EMT_scorehigh had a worse prognosis, and the IV_EMT_scorelow prognosis was similar to that of I_III group, which also explains the reason of difference in survival between groups in PD-L1 high-expression group. $* 00.05, * * \mathbb{\square}$ $0.01, * * * \otimes 0.001, * * \star \star \llbracket 0.0001$. 
a

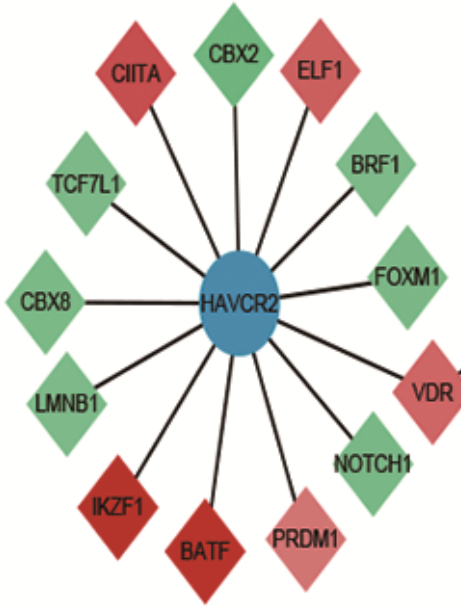

C

e

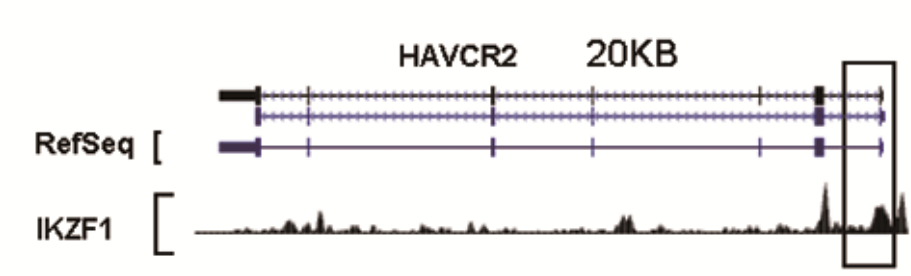

b

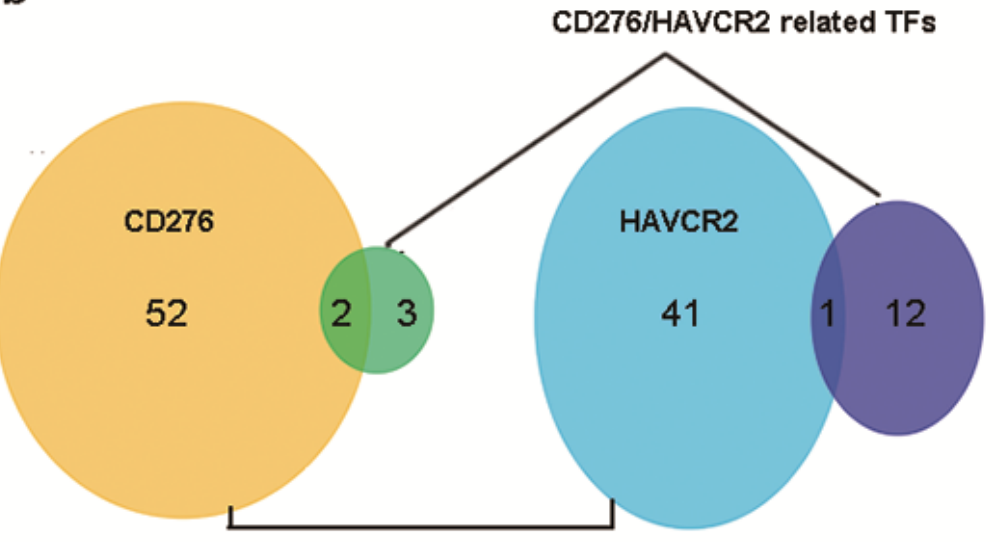

TFs that may bind to CD276/HAVCR2 by UCSC predicted

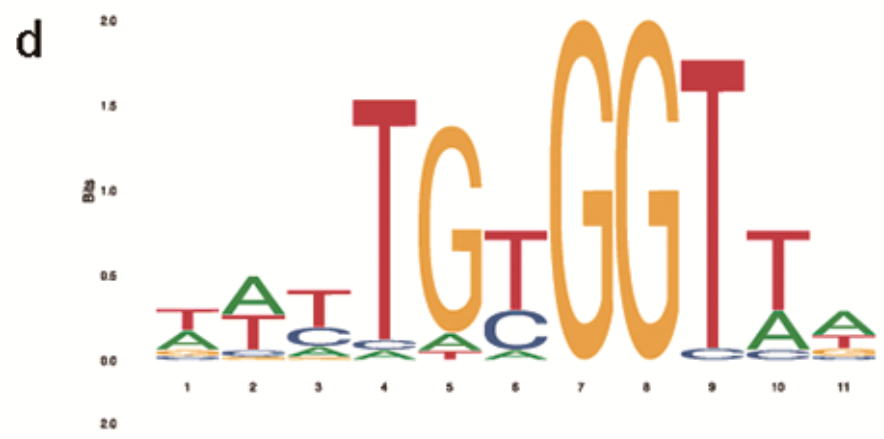

f $1:$

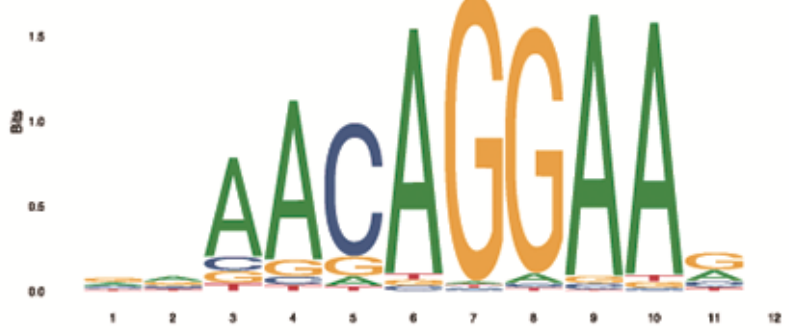

\section{Figure 11}

Finding CD276 and HAVCR2 upstream transcription factors. (A) Correlation analysis of differentially expressed tumor-associated transcription factors and CD276/HAVCR2, red for positive correlation, green for negative correlation, stronger correlation, darker color. (B) Intersection of transcription factors and associated transcription factors predicted by the UCSC database. (C and D) RUNX1 and CD276 possible binding peaks and corresponding binding sites predicted by UCSC database. (E and F) IKZF1 and HAVCR2 possible binding peaks and corresponding binding sites predicted by UCSC database.

\section{Supplementary Files}

This is a list of supplementary files associated with this preprint. Click to download.

- FigureS5.tif 
- Figures6.tif

- Figures4.tif

- Figures3.tif

- Figures7.tif

- FigureS1.tif

- FigureS2.tif 